\title{
LOS CIUDADANOS NO NACIONALES Y LOS IMPUESTOS LOCALES. EN LA PROVINCIA DE ALICANTE (1994-2014)
}

\author{
NON-NATIONAL CITIZENS REGARDING LOCAL TAXES IN ALICANTE \\ PROVINCE (1994-2014)
}

\author{
Irene Belmonte Martín \\ Universidad Miguel Hernández, Elche. España/Spain \\ irene.belmonte@umh.es
}

Recibido/Received: 11/08/2014

Aceptado/Accepted: 23/09/2014

\section{RESUMEN}

Este estudio ofrece una panorámica de la relación entre los extranjeros residentes en la provincia de Alicante -territorio con mayor presencia relativa de extranjeros en España- y los impuestos locales -la principal fuente de financiación de los municipios. Por un lado, se describe cómo las Administraciones Públicas Municipales, y en particular el organismo autónomo Suma Gestión Tributaria de la Diputación Provincial de Alicante, realizan el servicio de gestión y recaudación a sus ciudadanos extranjeros; por otro lado, se investiga la percepción de los extranjeros respecto a la forma en que se lleva a cabo este servicio público. Todo ello se sustenta en la idea que la plena integración de los extranjeros, como ciudadanos con sus derechos y obligaciones, fortalece sobremanera al gobierno local.

\section{PALABRAS CLAVE}

Impuestos locales, extranjeros, Suma Gestión Tributaria de la Diputación Provincial de Alicante, Servicio de atención al extranjero.

\section{SUMARIO}

1. Justificación del estudio. 2. Breve descripción de la Hacienda Local en España. 3. La panorámica de los extranjeros en la provincia de Alicante. 4. La atención al contribuyente extranjero por Suma Gestión Tributaria de la Diputación Provincial de Alicante. 5. La descripción de los resultados de las entrevistas realizadas a los contribuyentes extranjeros sobre la percepción de Suma. 6. Conclusiones. 7. Agradecimientos. Bibliografía

\begin{abstract}
This study offers an overview of the relationship between non-national citizens in the Alicante province - as the dominant province for foreigners -and their input to local taxes- as principal economic resource for municipalities. First, it aims to describe the strategies of the local public tax agency (Suma Gestion Tributaria de la Diputación Provincial de Alicante) to achieve better communication with non-national regarding the taxing system. Secondly, it explores the understanding of Suma's processes for the nonnational taxpayer. The motivation is that complete integration of foreigners, as citizens full of rights and duties, with mutual empowerment for the local government and citizens alike.
\end{abstract}

\section{KEYWORDS}

Local taxes, non-national citizens, Suma Gestión Tributaria de la Diputación Provincial de Alicante, foreigners Tax Service. 


\section{CONTENTS}

1. Justifying the study: Non-national citizens regarding Local Taxes. 2. Brief explanation of Local Taxes System in Spain. 3. An overview statistic of non- national in Alicante Province. 4. Foreigners Tax Service by Suma Gestión Tributaria de la Diputación Provincial de Alicante. 5. Interviews nonnational results. 6. Acknowledgements. 7. Conclusions. References.

\section{LA JUSTIFICACIÓN DEL ESTUDIO: LA IMPORTANCIA DE RELACIONAR LOS EXTRANJEROS A LA TRIBUTACIÓN LOCAL}

Los tributos locales (impuestos, tasas y precios públicos) constituyen junto con las transferencias del Estado, la principal fuente de financiación de los municipios (Ministerio de Hacienda y Administraciones Públicas. Presupuestos de las Entidades Locales 2013). Por todo ello, resultará fundamental para los municipios llevar a cabo una gestión tributaria de la forma más coordinada y completa, para dotar de cobertura económica a su Estado del Bienestar local. Y especialmente en un momento social como el actual, en el que repunta la pobreza y la desigualdad social, parece urgente reconciliar la democracia con el modelo económico del capitalismo a través de un "nuevo contrato social" que contemple, cuanto menos, restaurar la capacidad recaudatoria y equitativa de los sistemas fiscales, además de definir las prioridades del gasto público para construir una sociedad de oportunidades para los más débiles (Costas, 2014).

Por otra parte, según los datos del Instituto Nacional de Estadística (INE) en España viven alrededor de 5.000.258 extranjeros (según las cifras de población a 1 de enero de 2014, lo que viene a representar un 10,7 por ciento de los habitantes. www.ine.es, Fecha de consulta: 25/07/2014). Ciudadanos que de la misma forma que han de contar con sus derechos reconocidos también deben de estar al día de sus obligaciones tributarias para una plena integración en la sociedad española. Así, el presente trabajo, mediante el estudio de caso concreto de la provincia de Alicante, pretende describir, por una parte, las estrategias de la Administración Tributaria para extender las facilidades asociadas a la recaudación y gestión tributaria a los contribuyentes extranjeros al ser consciente de su diversidad social así como de su cultura tributaria diferenciada. Además cabría destacar el concepto de contribuyente extranjero va más allá del mero residente, pues tanto los residentes como los no residentes sin apenas relación con nuestro país salvo por poseer una propiedad, por ejemplo - pueden estar sujetos a pagar impuestos.

Por otra parte, este trabajo ofrece la perspectiva que tienen estos ciudadanos extranjeros sobre el modelo de tributación local en España y sus relaciones con la administración pública tributaria, a través de las entrevistas que para tal efecto se les ha realizado.

\section{BREVE DESCRIPCIÓN DE LA HACIENDA LOCAL EN ESPAÑA}

El actual sistema de financiación local, se encuentra establecido en el Real Decreto-Ley 2/2004, que regula la Ley de Haciendas Locales, si bien las Leyes de Presupuestos Generales del Estado de cada año en curso, suelen modificar puntualmente algunos de sus elementos. En este sentido, conviene recordar que las Haciendas Públicas Locales tienen, hasta cierto punto, limitada su capacidad de actuación aunque manteniendo su poder tributario, en el sentido que la falta de potestad legislativa de las Entidades Locales y, el sometimiento del 
tributo al principio de legalidad, determina que sea la ley estatal la que regule los aspectos esenciales del sistema tributario local; mientras que por otra, tienen margen para establecer los tipos de gravámenes y los beneficios fiscales que estimen aplicar a sus convecinos.

Tanto es así que los recursos de las Haciendas Locales provienen de los ingresos que generan sus propios tributos, las transferencias que reciben del Estado, y de las Comunidades Autónomas, así como de otros ingresos de origen vario.

Tabla 1. Los principales ingresos de los municipios

\begin{tabular}{|l|l|l|}
\hline $\begin{array}{l}\text { Ingresos de } \\
\text { origen tributario }\end{array}$ & Impuestos & Obligatorios: IBI, IAE, IVTM \\
\cline { 2 - 3 } & Tasas & $\begin{array}{l}\text { Potestativos: IIVTNU, ICIO } \\
\text { alcantarillado, suministro, etc. }\end{array}$ \\
\cline { 2 - 3 } & $\begin{array}{l}\text { Contribuciones } \\
\text { Especiales }\end{array}$ & $\begin{array}{l}\text { Contribuciones como consecuencia de } \\
\text { mejoras realizadas por la Administración } \\
\text { Local e incremento del valor. }\end{array}$ \\
\hline Transferencias & $\begin{array}{l}\text { Participación en los ingresos del Estado (PIE) y participación en } \\
\text { los ingresos de las Comunidades Autónomas (PICA) }\end{array}$ \\
\hline $\begin{array}{l}\text { Ingresos no } \\
\text { tributarios }\end{array}$ & Ingresos procedentes de su patrimonio de derecho privado \\
\cline { 2 - 3 } & Precios públicos \\
\cline { 2 - 3 } & Operaciones de crédito \\
\cline { 2 - 3 } & Multas y sanciones en el ámbito de sus competencias \\
\cline { 2 - 3 } & Subvenciones \\
\hline
\end{tabular}

Fuente: Elaboración propia a partir del RDL 2/2004 que regula la LHL.

Los ingresos de origen tributario están constituidos por los impuestos, las tasas y las contribuciones especiales. Los cinco impuestos locales a su vez son: el Impuesto de Bienes Inmuebles (IBI), el Impuesto de Actividades Económicas (IAE), el Impuesto de Vehículos de Tracción Mecánica (IVTM), el Impuesto de Incremento de Valor de los Terrenos de Naturaleza Urbana (IIVTNU, lo que se conoce como Plusvalía) y, el Impuesto de Construcciones y Obras (ICIO). Las tasas son las contribuciones económicas, que realizan los usuarios de un servicio público y sólo se hacen exigibles en el caso que el ciudadano decida utilizar ese servicio, con objeto de recuperar total o parcialmente el coste financiero del mismo (como la tasa de recogida de basuras, la de extinción de incendios, aquellas por la emisión de certificados, etc.). Por otra parte, las contribuciones especiales, son unos tributos que gravan el beneficio o de un aumento de valor de los bienes como consecuencia de la realización de obras públicas o del establecimiento o ampliación de servicios públicos. En la actualidad, su exigencia por el Ayuntamiento es voluntaria y, precisa de un acuerdo de imposición y la correspondiente Ordenanza Fiscal.

Los recursos que provienen de las transferencias engloban tanto la cesión de la recaudación de los impuestos del Estado, la participación en los tributos estatales (como el IRPF o el IVA) y la participación de los tributos propios de las Comunidades Autónomas en la forma y cuantía que determinen las leyes de sus propios Parlamentos.

El resto de ingresos de los municipios son aquellos que proceden de la explotación de su patrimonio y demás actividades de derecho privado, del producto de las operaciones de crédito (endeudamiento), de las multas y sanciones que éstos puedan imponer en el ámbito de sus competencias, de las subvenciones, y, por último, del uso de los precios públicos.

Por otra parte, la Constitución de 1978 otorga a los municipios la autonomía y suficiencia financiera (arts. 137 y 142), pero sin los suficientes recursos, éstos no podrán hacerse 
efectivos. Además, los municipios juegan un papel fundamental en el desarrollo y mantenimiento del Estado del Bienestar, en base a los principios de subsidiariedad y proximidad. Razonablemente, el Estado del Bienestar local sólo se puede sostener con un equilibrado volumen de ingresos públicos que se ajuste a los previsibles gastos sociales, por lo que resultará fundamental la optimización de dichos beneficios.

Dada la complejidad asociada a la gestión tributaria local en nuestro país, la mayor parte de los municipios han delegado la gestión de sus tributos en una organización supramunicipal, siendo ésta la Diputación Provincial, mientras que a su vez las Diputaciones Provinciales también tienden a constituir organismos autónomos ad hoc para la recaudación y gestión de sus tributos (Belmonte, 2013). Este sería el caso de la provincia de Alicante, y así en 1993, creó el organismo autónomo Suma Gestión Tributaria de la Diputación Provincial de Alicante, en adelante simplemente Suma, como modelo de Modernización Administrativa de la gestión tributaria local. La misión principal de este organismo es la gestión y la recaudación de los tributos y otros ingresos de derecho público de los Ayuntamientos y otras Administraciones, mediante convenio de delegación.

Resulta especialmente destacable el hecho diferenciador en la panorámica nacional que todos, los 141 Ayuntamientos alicantinos, hayan delegado la gestión de sus tributos o ingresos públicos en Suma. Esta circunstancia, cobra aún más relevancia dadas las particularidades de la provincia alicantina, al ser la cuarta provincia más poblada del país (tras Madrid, Barcelona y Valencia), con un porcentaje de municipios de más de 50.000 habitantes que septuplica la media nacional y con el más alto porcentaje de población extranjera, pues el 24,12 por ciento de los residentes alicantinos son extranjeros, conforme a los datos del INE, Cifras de población a 1 de enero de 2013, resultados definitivos (www.ine.es; Consulta:26/07/2014).

\section{LA PANORÁMICA DE LOS EXTRANJEROS EN LA PROVINCIA DE ALICANTE}

Como muestran los datos que aporta el INE, la presencia de extranjeros en la provincia de Alicante 469.417 personas, no sólo es muy fuerte a nivel de relativo sino que, en términos absolutos, tan sólo es superada por las provincias de Madrid ( 960.121 personas y 14,78 por ciento de extranjeros) y Barcelona (771.990 personas que representan el 13,93 por ciento). Tanto es así que el 52,84 por ciento de todos los residentes extranjeros censados en España en el año 2013, se concentran en estas tres provincias, lo que supone un total de 2.201.528 personas.

En cualquier caso, y a pesar de la fuerte presencia, la población extranjera está decayendo en los últimos años a nivel nacional. Así, durante el año 2013, el número neto de españoles inscritos experimenta un aumento de 141.361 personas ( 0,3 por ciento), mientras que el de extranjeros desciende en 545.980 ( $-9,8$ por ciento). Entre estos últimos, los pertenecientes a la UE-28 disminuyen en 313.446 (hasta un total de 2.047 .532 personas), mientras que los no comunitarios se reducen en 232.534 personas (INE, Nota de prensa, 22 de abril de 2014). 
Tabla 2. La población extranjera y la población total en las provincias españolas según importancia relativa (a 1 de enero de 2013).

\begin{tabular}{|c|c|c|c|}
\hline Provincia & Extranjeros & Total población & Porcentaje \\
\hline 1. Alicante & 469.417 & 1.945 .642 & 24,12 \\
\hline 2. Girona & 161.025 & 761.632 & 21,14 \\
\hline 3. Almería & 146.656 & 699.329 & 20,97 \\
\hline 4. $\quad$ Lleida & 81.145 & 440.915 & 18,40 \\
\hline 5. Castellón & 108.443 & 601.699 & 18,02 \\
\hline 6. Málaga & 296.337 & 1.652 .999 & 17,92 \\
\hline 7. Tarragona & 144.312 & 810.178 & 17,81 \\
\hline 8. $\quad$ Murcia & 231.022 & 1.472 .049 & 15,69 \\
\hline 9. $\quad$ Guadalajara & 39.368 & 257.723 & 15,27 \\
\hline 10. Madrid & 960.121 & 6.495 .551 & 14,78 \\
\hline
\end{tabular}

Fuente: Elaboración propia a partir del Instituto Nacional de Estadística (www.ine.es: Consulta: 26/07/2014)

Sin embargo, en la provincia de Alicante, la población extranjera se mantiene en torno a las mismas cifras de los años del comienzo de la crisis económica. La evolución de la población extranjera en Alicante se refleja en la figura 2. Nótese que tanto el Observatorio Valenciano de la Inmigración como el INE, sólo disponen de datos de la inmigración en España a partir del año 1997.

Figura 1. Evolución de la población extranjeros en la provincia de Alicante (1997-2013)

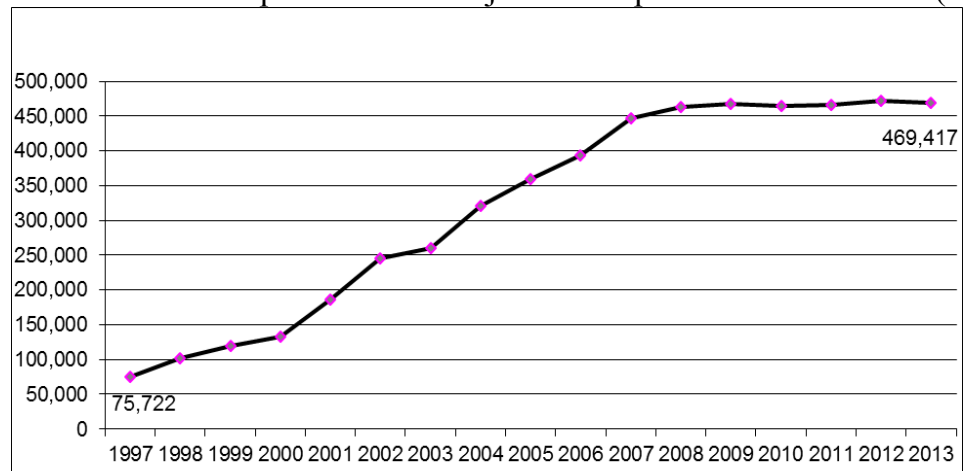

Fuente: Elaboración propia a partir de las cifras del INE (Fecha de consulta: 26/07/2014).

Por otra parte, también resulta destacable que las localidades españolas con mayor proporción de extranjeros son alicantinas, tanto en los municipios pequeños como en las ciudades medianas. De hecho, en diecinueve municipios alicantinos (que constituyen casi el 14 por ciento del total de ellos), la población extranjera supera a la nacional. El municipio alicantino de San Fulgencio es la localidad española con más extranjeros, en la que el 77,58 por ciento de sus 12.144 habitantes no son españoles. Asimismo es reseñable todos los municipios españoles de más de 10.000 habitantes en los que los extranjeros superan a los nacionales son alicantinos (San Fulgencio, Rojales, Teulada, Calpe, Alfas del Pí, Jávea y Torrevieja). Por otra parte, Torrevieja es la ciudad española, de más de 50.000 habitantes, con mayor presencia no autóctona, que representa un 52,76 por ciento del total. Además estimamos que estos datos oficiales pueden estar subestimando la realidad en la medida que 
los extranjeros constituyen una parte importante de la población flotante de la provincia que no está reflejados en ningún tipo de padrón municipal.

Tabla 3. Distribución de población extranjera por municipios en la provincia de Alicante.

\begin{tabular}{|l|c|c|}
\hline $\begin{array}{l}\text { Población extranjera } \\
\text { en Alicante } 2010\end{array}$ & $\begin{array}{c}\text { Número } \\
\text { Municipios }\end{array}$ & $\begin{array}{l}\text { Número Municipios con población } \\
\text { extranjera superior al 50 por ciento }\end{array}$ \\
\hline $\begin{array}{l}\text { Menos de 5.000 habitantes } \\
\text { (Pequeños municipios) }\end{array}$ & 83 & 10 \\
\hline $\begin{array}{l}\text { Entre 5.000 y 20.000 } \\
\text { (Municipios medianos) }\end{array}$ & 34 & 4 \\
\hline $\begin{array}{l}\text { Entre 20.001 y 50.000 } \\
\text { (Pequeñas ciudades) }\end{array}$ & 16 & 1 \\
\hline $\begin{array}{l}\text { Entre 50.001 y 200.000 } \\
\text { (Ciudades medianas) }\end{array}$ & 6 & 0 \\
\hline $\begin{array}{l}\text { Más de 200.000 } \\
\text { (Grandes ciudades) }\end{array}$ & 2 & 19 \\
\hline TOTAL & 141 & 4 \\
\hline
\end{tabular}

Fuente: Observatorio Valenciano de la Inmigración (http://www.observatorioinmigracion. gva.es: Consulta. 22/05/2014)

Así, los pequeños municipios (aquellos con una población inferior a los 5.000 habitantes) con un porcentaje de población extranjera superior al 50 por ciento son: Alcalalí $(61,09$ por ciento), Algorfa (76,73 por ciento), Daya Vieja (68,68 por ciento), Hondón de los Frailes (52,50 por ciento), Murla (52,78 por ciento), Orba (56,68 por ciento), Ràfol de Almunia (51,65 por ciento) y Poblets (62,63 por ciento). Los municipios medianos (entre 5.000 y 20.000 habitantes): Benitaxell (70,46 por ciento) San Fulgencio (77,58 por ciento), San Miguel de Salinas (64,37 por ciento) y Teulada (66,51 por ciento). Las pequeñas ciudades (entre 20.000 y 50.000 habitantes): Benitaxell (70,46 por ciento) San Fulgencio (77,58 por ciento), San Miguel de Salinas (64,37 por ciento) y Teulada (66,51 por ciento). Y, por último la ciudades mediana, de Torrevieja con 102.136 habitantes y el 52,76 por ciento de extranjeros (Datos del Observatorio Valenciano de la Inmigración 2011).

\section{LA ATENCIÓN AL CONTRIBUYENTE EXTRANJERO}

La elevada proporción así como particularidades de los ciudadanos extranjeros en la provincia de Alicante, abren el camino a que la Agencia Tributaria de la Diputación Provincial que se encarga de la gestión y recaudación de los tributos, Suma, deba realizar una actividad especial tanto para atender a este tipo de contribuyentes con unas características especiales (de idioma, con menor conocimiento del funcionamiento del sistema tributario o cultura tributaria diferente y/o en situación de temporalidad) (Bevins y Franks, 2003: 83) como para evitar evasiones de sus obligaciones como ciudadanos.

Las cualidades y, en algunos casos, las diferencias lingüísticas, culturales y sociales, de los extranjeros, unidas al hecho que gran parte de estos colectivos no tienen un arraigo laboral y temporal permanente en la provincia, impulsaron al organismo tributario a poner en marcha unos servicios específicos conforme a sus peculiaridades.

Así, en el año 1994, Suma pone en funcionamiento la Unidad de Atención a Contribuyentes Extranjeros, con el objetivo inicial de divulgar entre éstos la información tributaria y facilitarles el cumplimiento de sus obligaciones con la administración. El devenir 
de los años, no sólo ha vislumbrado un espectacular crecimiento de la población extranjera en nuestra provincia, como fiel reflejo de la realidad nacional (tal y como refleja la figura 1), sino que también ha expandido los servicios de Suma, extendiendo la actividad del servicio de atención a extranjeros desde la meramente informativa y divulgativa a una actitud proactiva hacia este colectivo.

"Lo más característico y excepcional del caso español ha sido el cambio radical de una sociedad de emigración a un país de inmigración en un plazo de tiempo muy reducido" (Garrido, 2008: 129). En general, la velocidad con la que han cambiado las coordenadas del fenómeno de la inmigración -hasta prácticamente el año 2010 en que la recesión económica aplica su realidad con más crudeza a los inmigrantes imponiendo un retorno a sus países de origen- no logró conseguir la consolidación de un modelo de inmigración que integrara una legislación sólida, una respuesta política coherente y una adaptación social eficiente (Garrido, 2008: 163). Por este motivo, quisiéramos hacer constar que el servicio que lleva a cabo Suma como una acción integradora hacia la inmigración en lo que respecta al cumplimiento de sus deberes tributarios. Pues este servicio, que facilita la gestión y comprensión del sistema español a los no-nacionales, supone una acción de integración de este colectivo, máxime considerando que los residentes extranjeros tratan mayormente de cumplir con sus obligaciones tributarias en nuestro país, tal y como acreditan las entrevistas que se les ha realizado.

En este sentido, los servicios de atención a contribuyentes extranjeros, evidentemente, han de tomar en consideración la distribución de la población foránea a fin de focalizar las actuaciones de los colectivos con más representación en la provincia. El colectivo más numeroso lo conforman los ciudadanos de la UE, resultando que el 72,30 por ciento de los extranjeros en Alicante son de origen europeo (según los datos del INE para 2013). Las razones de la arraigada presencia de los ciudadanos europeos se podrían resumir en: el denominado estilo de vida que se concreta en la expectativa de una mejor calidad de vida (clima y salud), los factores geográficos y las condiciones económicas (la vivienda como inversión y el menor coste de la vida). De la deducción de estas razones, se comprende el hecho que exista un importante del colectivo de ciudadanos en situación de inactividad asentados en la provincia alicantina (Huete, 2005: 382-387). En cuanto al resto de ciudadanos por continentes, destacan los americanos (12,51 por ciento con importante presencia de los latinoamericanos especialmente) y los africanos (11,73 por ciento). Si bien se estima que la razón principal de estos colectivos para asentarse en nuestro país es el trabajo (García, 2010).

En lo que respecta al colectivo mayoritario, los extranjeros europeos, el estilo de vida que desarrollan cuando eligen la provincia de Alicante para trasladarse, puede describirse a partir de dos patrones: para el primer grupo, paz, relax y vida rural; y fiesta, sol y playa, para el segundo grupo. Dependiendo de qué tipo de características predomine, eligen una determinada zona para asentarse en función de un conocimiento previo o referencias de sus compatriotas. Complementariamente, los estilos de residencia de los extranjeros son la combinación de ciclo vital y nacionalidad. Así pueden diferenciarse en función del estilo de vida o función que reproduzcan en nuestro territorio: por una parte, el residente que busca la participación e integración en la vida social y laboral; y por otra, el jubilado o trabajador estacional, que se retira para mejorar su calidad de vida o descansar, tendente a formar colonias y guetos (Huete, 2005: 388). 
Figura 2. La distribución de la población extranjera por continentes en la provincia de Alicante.

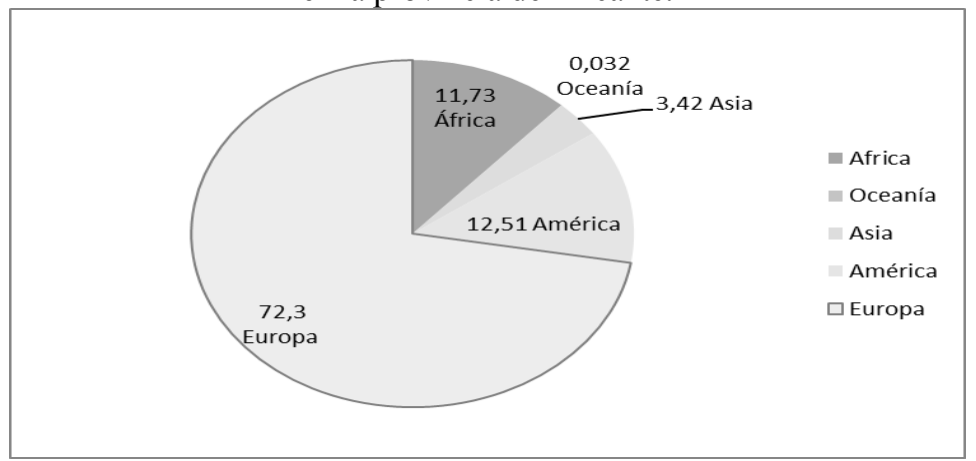

Fuente: Elaboración propia a partir de las cifras del INE (Fecha de consulta: 26/07/2014).

En cuanto a las principales nacionalidades, destaca la presencia de británicos $(27,80$ por ciento del total de extranjeros), marroquíes ( 7,81 por ciento), alemanes ( 7,65 por ciento), rumanos (7,25 por ciento), colombianos ( 4,8 por ciento) y ecuatorianos (3,2 por ciento) (Fuente: INE con datos de 2013). En este sentido, las cifras que arroja el Observatorio Valenciano de la Inmigración muestran que la sociedad alicantina se ha convertido en un crisol de culturas, en la que conviven 122 nacionalidades y se hablan más de 40 lenguas diferentes (Núñez, 2013: 77).

Figura 3. La distribución de la población extranjera por nacionalidades en la provincia de Alicante.

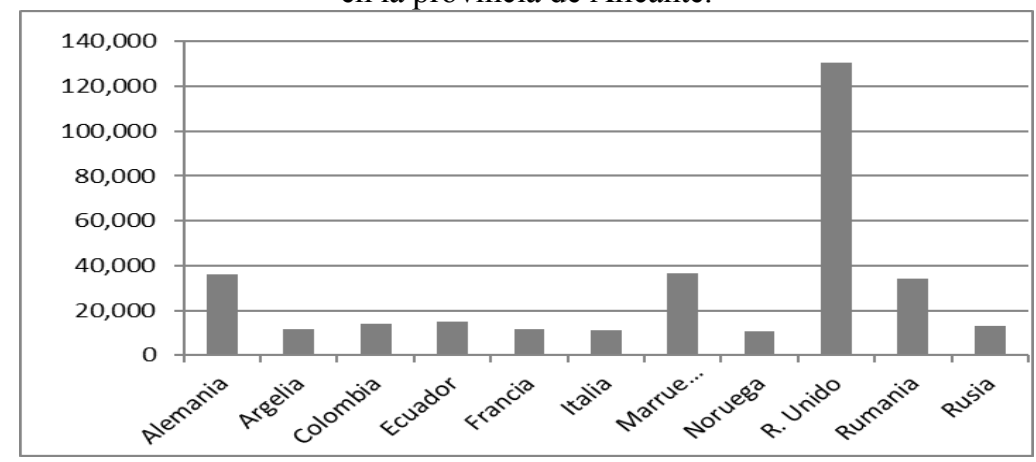

Fuente: Elaboración propia a partir de las cifras del INE (Fecha de consulta: 26/07/2014).

Resulta cuanto menos curioso evidenciar algunos aspectos sobre el grado de integración del colectivo de los británicos. Así, el $78 \%$ reconoce que se relaciona sólo con ciudadanos ingleses, porque no existen españoles en su zona de residencia (52\%) ni en sus hábitos sociales y familiares (48\%). Además también manifiestan consumir con asiduidad los medios de comunicación ingleses en lugar de los españoles (González Sanz, 2011: 15-16).

Dada la dualidad que existe entre extranjeros con arraigo o no en nuestro país, también el servicio de atención al contribuyente extranjero de Suma, distingue entre las actuaciones a realizar para los residentes como los no residentes (López Mingueza, 2009) 
- Las actuaciones hacia los residentes:

a) Atención en idiomas: para lo que existe la unidad de extranjeros con capacidad para atender en inglés, alemán, francés, y holandés. Esta atención en idiomas no solo se centra en las oficinas de Suma repartidas geográficamente sino que también se extiende a los servicios telefónicos (Centro de Atención al Usuario) y por correo electrónico. Precisamente el 34,18 por ciento de las consultas por email que recibió Suma durante el año 2013 correspondieron a ciudadanos extranjeros (Memoria Suma 2013).

Además Suma presta especial importancia a la formación de su personal en idiomas.

Se hace constar que Suma no ofrece atención ni información en el idioma ruso, siendo esta colectividad muy importante en la provincia alicantina y además en continuado aumento, especialmente en la zona sur que corresponde con Torrevieja.

b) Publicación de la información tributaria municipal, en los distintos idiomas a través de las asociaciones y prensa especializada para extranjeros. Entre la prensa extranjera en la que Suma publica los anuncios se destacan: Costa Blanca News, Costa Blanca Nachrichten, Think in Spain, Wochen Post, La Gaviota, Round Town News y Coast Rider. Los colectivos asociativos corresponden a las nacionalidades holandesas, francesas, alemanas, belgas, inglesas, noruegas y suizas (López Mingueza, 2009).

c) Se realizan también sesiones informativas, como las organizadas con los colectivos extranjeros que de alguna manera se encuentran agrupados en colectivos y asociaciones como por ejemplo, El Campello English Speaking Club.

d) En la Web toda la información se encuentra disponible en todos los idiomas que permite el traductor de Google además, diversos archivos publicados también están traducidos al inglés, alemán y francés. La versión inglesa de la página oficial de Suma, se encuentra actualizada.

e) Por ultimo cabría resaltar que la destacada presencia de residentes británicos ha impulsado la colaboración entre Suma y el Instituto de Revenues Rates and Valuation (IRRV), institución profesional de técnicos tributarios del Reino Unido, para buscar las claves que comprendan mejor el funcionamiento de nuestro sistema tributario local por los extranjeros.

Esta colaboración contempla una doble perspectiva, por una parte, el personal de Suma forma a técnicos británicos en España para facilitar el pago en su país de los hechos imponibles que se producen en nuestra provincia; mientras que por otra, los técnicos de Suma observan in situ y con detalladas explicaciones de los técnicos británicos cómo funciona su sistema tributario local para comprender mejor la idiosincrasia del contribuyente británico. La puesta en práctica de este proyecto se ejecuta a través del programa Leonardo de la Unión Europea (Andarias, 2012 y 2013).

El proyecto Leonardo Da Vinci, es un programa de la UE para promocionar la cultura europea en el ámbito laboral. Suma junto con el citado IRRV, ha participado ya en la iniciativa de formación que promueve las estancias trasnacionales entre empresas para personas en el mercado laboral (PLM, "People in the labour market"). La convocatoria que realizaron ambos organismos corresponde a la siguiente iniciativa: ES/10/LLP/LdV/PLM/ 150124 .

- Las actuaciones dirigidas a los contribuyentes no residentes:

a) La localización de deudores. Los contribuyentes no residentes pasan al estadio de localización de deudores, cuando confluyen los siguientes requisitos: 1. La deuda ha de estar en período ejecutivo; es decir, cuando se haya superado el periodo voluntario de pago, 2 . No tienen designado representante en España; 3. Se les ha sido practicadas las pertinentes notificaciones en su domicilio fiscal, y; 4. Se han agotado todas las posibles vías de localización en el territorio nacional. Los trabajos de localización implican averiguar el 
nuevo municipio o país de residencia del contribuyente a través de la documentación de cambio de titular o posibles movimientos de domiciliaciones bancarias $\mathrm{y}$, también, localizar su dirección a partir de la información que pueda suministrar Internet o los propios Ayuntamientos (López Mingueza, 2009). Suma ha desarrollado específicamente un proyecto para localizar británicos, por ser el colectivo de la UE más numeroso, a partir de la colaboración con una empresa británica que localiza a los contribuyentes (Rossendales).

b) La elaboración de modelos de comunicación en inglés, alemán y francés para el requerimiento del pago de la deuda y el aviso del inicio del procedimiento de embargo.

c) Las facilidades para realizar otros medios de medios de pago como: a través de transferencia bancaria, telefónicamente con tarjeta de crédito y con domiciliación (SEPA), además de las vías de pago tradicional.

d) Otro proyecto es la videoconferencia, destinado a la formación y seguimiento continuo del proyecto formativo de recaudación de los tributos españoles en territorio extranjero.

Recapitulando, los extranjeros suponen un colectivo de contribuyentes importante que, en general, cumplen con sus obligaciones tributarias con formalidad. Si bien, en muchas ocasiones, el carácter no residencial y su grado de impermeabilidad al desarrollo de la sociedad española, hacen necesario que los grupos sean atendidos de forma específica. Así, el organismo tributario Suma no sólo ha de contemplar ampliamente las actuaciones particulares descritas, sino que también deberá tomar esas consideraciones, de manera más específica, en las oficinas presenciales sitas en las comarcas y municipios en los que estos extranjeros se suelen concentrar.

\section{LA DESCRIPCIÓN DE LOS RESULTADOS DE LAS ENTREVISTAS REALIZADAS A LOS CONTRIBUYENTES EXTRANJEROS SOBRE LA PERCEPCIÓN DE SUMA}

La técnica cualitativa de la entrevista, es considerada como un procedimiento científico para obtener información cualificada (González Río, 1997:166). Las entrevistas tratan de conseguir datos discursivos, lo que quiere decir que recogen el discurso completo de los actores sociales identificados como relevantes, para describir la estructura significativa de un asunto en concreto (Uña et al., 2009: 84). En este sentido, se han seleccionado 21 entrevistas realizadas a diversos contribuyentes extranjeros en la provincia de Alicante. De estas, 14 corresponden al colectivo de británicos, 3 a noruegos y 3 a italianos. Estas seis últimas han sido especialmente seleccionadas atendiendo al criterio que no son hablantes nativos de lenguas mayoritarias en la UE pero que sin embargo tienen una importante presencia en el área geográfica. Entre los entrevistados, 11 son mujeres y 10 hombres. Y la estructura de edad se distribuye de la siguiente forma:

Tabla 4. Distribución de los entrevistados por grupos de edad

\begin{tabular}{|c|c|}
\hline Intervalo de edad & $\begin{array}{l}\text { Número de } \\
\text { entrevistados }\end{array}$ \\
\hline 25 a 45 años & 4 \\
\hline 46 a 64 años & 6 \\
\hline 65 o más & 11 \\
\hline
\end{tabular}


Además, se ha atendido al criterio de población residente y no residente en nuestro país, considerando a éstos últimos aquellos que están en nuestro país menos de 1 año.

Tabla 5. Distribución de los entrevistados según el tiempo que llevan en España

\begin{tabular}{|l|l|}
\hline Residente: más de 1 año en España & $16(76,2$ por ciento $)$ \\
\hline Menos de 1 año & $5(23,8$ por ciento $)$ \\
\hline
\end{tabular}

La recogida de datos se ha realizado durante el mes de junio de 2014, básicamente por los usuarios del "English Speaking Club de El Campello" que agrupa a los extranjeros de las comarcas del Alicantí y la Marina Baja.

La modalidad de entrevista ha sido la semiestructurada, pues incluye diferentes tipos de preguntas (cerradas, abiertas y de confrontación) sobre distintos aspectos que la entrevistadora ha considerado de interés, y se complementa, con ideas sobre cómo estructurar su contenido durante la recogida de datos (Flick, 2004: 104).

Así, el cuestionario de base para las entrevistas realizadas a los extranjeros sobre la percepción de la satisfacción de los servicios de gestión tributaria y recaudación que realiza Suma Gestión Tributaria de la Diputación Provincial de Alicante, ha sido diseñado en inglés, y se expone a continuación.

Tabla 6. El cuestionario elaborado para los contribuyentes extranjeros This very short questionnaire is being carried out to assist research which should prove vital in improving Suma services.

Thank you very much for taking part!!

Question 1

Paying Suma is

a) simple b) complicated c) no opinion d) not applicable

Question 2

Who is Suma?

a) local council b) civil service c) regional government

d) provincial government e)do not know

Question 3

Have you seen any Suma literature in English, French or German?

a) yes b) no c) do not know

Question 4

In the Suma office the service is

a) good b) average c) poor d) third party e) not applicable

Question 5

By telephone with Suma the service is

a) good b) average c) poor d) third party e) not applicable

Question 6

Do you pay by monthly installments?

a) yes b) no c) not aware of this option

Your answers are anonymous but it would be useful to know

1. your age

2. your nationality

3. how long you have been living in Spain

Descripción de los resultados 
La mayor parte de los usuarios consideran que su relación con Suma es sencilla. Y coinciden aquellos que contestan que no es aplicable con aquellos extranjeros que llevan menos de un año en el país, con lo que se estima que no son, ni han sido, sujetos pasivos de los tributos locales.

Pregunta 1: Paying Suma is

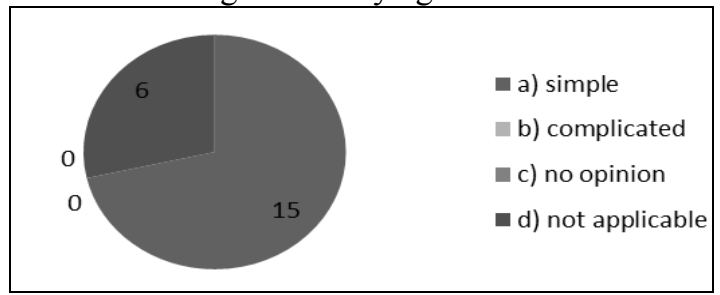

En cualquier caso, el $15 \%$ de los entrevistados reconoce que ha tenido alguna vez un problema administrativo, como emisión de recibos indebidos, errores en los recibidos emitidos o bien la dirección postal de aviso era inexacta. Estas personas manifiestan que la resolución de estas incorrecciones les llevó mucho tiempo y esfuerzo.

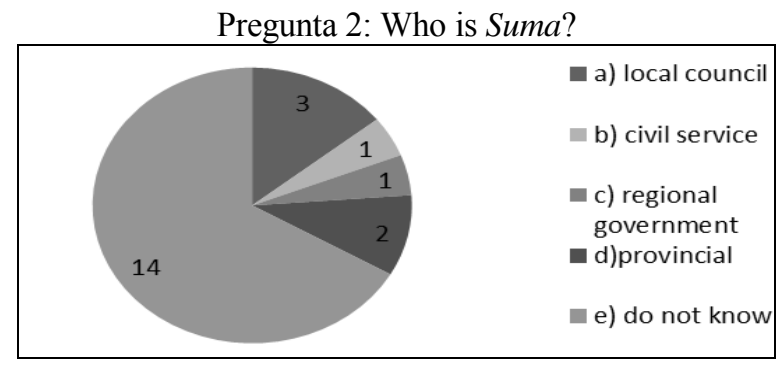

Tan sólo 2 entrevistados aciertan a la hora de identificar que Suma es un organismo autónomo de la Diputación Provincial de Alicante a pesar de la intensa publicidad al respecto. Esto es una muestra de que la complejidad de nuestro sistema administrativo (que combina el nivel local con el provincial, regional y nacional) apenas es entendida por los extranjeros.

Pregunta 3: Have you seen any Suma literature in English, French or German?

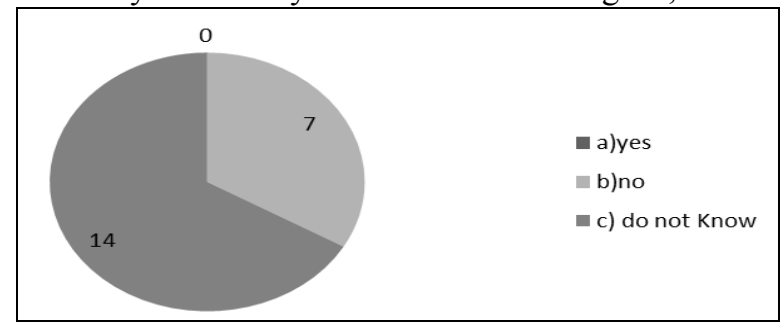

Resulta curioso destacar que ninguno de los entrevistados conocía de la publicidad de Suma en otros idiomas. De hecho, algunos reconocieron que la información sobre el pago del IVTM la obtuvieron en el taller de reparación de su coche. 
Pregunta 4: In the Suma office the service is

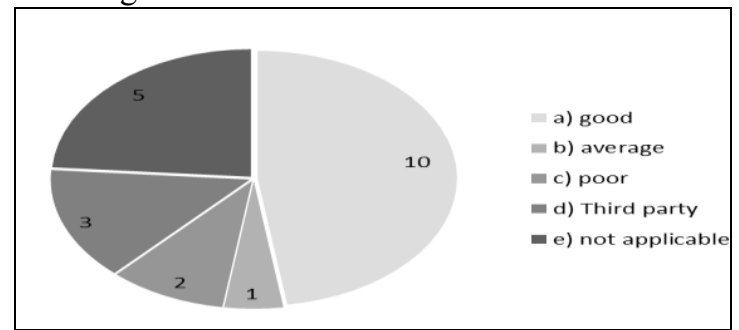

La mayor parte de los entrevistados reconocen la buena calidad del servicio de atención en las oficinas de Suma. En cualquier caso, resulta destacable que 3 entrevistados utilicen a una tercera persona para realizar sus trámites relacionados con la gestión tributaria local. En este sentido, mientras un entrevistado reconoce que si hubiera sabido que en las oficinas se hablaba inglés no hubiera acudido a una tercera persona, otra persona reconoce que tuvo que acudir a un traductor pues no conseguía entender ni hacerse entender con el personal de Suma "I was unable to solve the matter myself despite having lived here for 30 years and with what I thought was a reasonable level of the Spanish language" (transcripción literal de la entrevista)

Pregunta 5: By telephone with Suma the service is

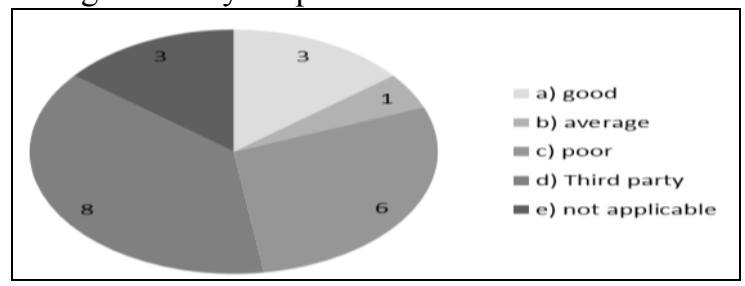

Precisamente es la cuestión de la atención no presencial en la que el 76,2 por ciento de los entrevistados (14 personas) reprenden la actividad desarrollada por Suma, al calificarla de baja calidad y precisar de la ayuda de una tercera persona para requerir la información telefónica. En cualquier caso, también 2 entrevistados reconocen que la persona de atención telefónica hablaba muy bien inglés y su disposición era muy positiva, aunque no estaba autorizada para realizar modificaciones y que precisaba de una autorización, lo que obligaba al contribuyente a tener que repetir el trámite.

Pregunta 6: Do you pay by monthly installments?

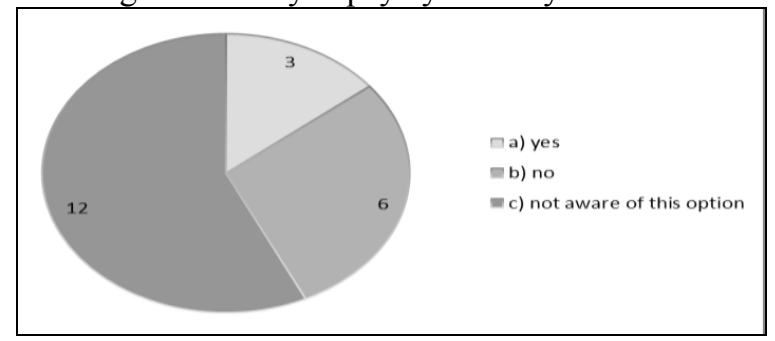


Apenas el $14 \%$ de los entrevistados utilizan el plan de pago personalizado de Suma que permite el hacer frente de las obligaciones tributarias de los contribuyentes de forma fraccionada y organizando los pagos según su conveniencia. Resulta significativo que algo más el 57\% no conociera este servicio que presta Suma y forma parte de la idiosincrasia de su modelo de gestión. Algunos entrevistados se sorprenden que existan folletos en inglés explicando en qué consiste el Plan de Pago Personalizado.

\section{CONCLUSIONES}

1. Los tributos constituyen la principal fuente de financiación para los municipios, entre los que tiene especial protagonismo el IBI. Por otra parte, España es un destino para muchos extranjeros, siendo algo más de un $10 \%$ de su población reconocida en el INE como extranjera, resultando por lo tanto como sujetos pasivos de los tributos.

2. En particular, al ser Alicante la provincia española con mayor presencia relativa de extranjeros (alrededor de una cuarta parte según cifras las oficiales de su población), resultará fundamental establecer una serie de estrategias desde la Administración Pública para hacer comprender a este colectivo la idiosincrasia del modelo de tributación local. Entre los extranjeros destaca la fuerte presencia de ciudadanos británicos.

3. El organismo autónomo de la Diputación Provincial de Alicante, Suma Gestión Tributaria, se encarga de la gestión tributaria y recaudación de los tributos locales y otros ingresos de derecho público por delegación de "todos" los municipios alicantinos. Así, ha establecido una serie de actuaciones dirigidas especialmente hacia el colectivo de los no-nacionales a través de su Unidad de Atención de Contribuyentes Extranjeros. Medidas que abarcan desde la atención, información y publicidad en varios idiomas europeos, convenios de colaboración con empresas especializadas en gestión tributaria del Reino Unido, etc.

4. La información que se ha recabado de las entrevistas realizadas a los extranjeros nos muestran que son un colectivo que desea cumplir puntualmente con sus obligaciones tributarias en España, aunque en algunos aspectos, y a pesar, de las medidas emprendidas por Suma, les resulta difícil comprender nuestra cultura tributaria. Siendo consciente de las limitaciones asociadas a este primer estudio experimental, no descartamos desarrollar investigaciones y exploraciones futuras para ahondar más en este tema.

\section{AGRADECIMIENTOS}

Quisiera hacer constar en este breve apartado, mi agradecimiento a las 21 personas que de forma desinteresada han dedicado parte de su tiempo de ocio y asueto para dar respuesta a este cuestionario, pues sus comentarios aportan más sentido y valor a este trabajo.

Además de manera especial, quisiera dejar patente mi agradecimiento a Miss Heather Abley, investigadora social y amiga entrañable, por su extraordinaria visión en la elaboración del cuestionario y por su tenacidad en el tratamiento de la información que ha recogido de los extranjeros en Alicante. 


\section{BIBLIOGRAFÍA}

ANDARIAS, R. (2012). "The return match-played on a UK pitch 1". En: Insight, March . www.irrv.net. ANDARIAS, R. (2013). "Crónica de una visita profesional de técnicos tributarios de Suma al Reino Unido, algo más que un proyecto común de movilidad”. En: Revista de Estudios Locales, 154: 8-11.

BELMONTE, I. (2013). "La gestión tributaria local en España: Algunas propuestas solidarias del estado del bienestar ante la crisis global". En: Barataria. Revista Castellano Manchega de Ciencias Sociales, 15: 39-55.

BLEVINS, B. y FRANKS, D. (2003). "Local Spanish Taxes and miscellaneous". En: Living in Spain. London: Blevins Franks, pp. 81-88.

COSTAS, A. (2014). "Capitalismo, desigualdad y democracia”. En: El País Negocios, 20 de julio, p.15. FLICK, U. (2004). Introducción a la investigación cualitativa. Madrid: Morata.

GARCÍA, I. (2010). "Políticas migratorias en España y cooperación con los países de América Latina". En V. Benito y A. L. Guzmán (coords.). Ponencias, comentarios y conclusiones del I Seminario Iberoamericano sobre Políticas Migratorias, Cooperación al Desarrollo, Interculturalidad e Integración Social de los Inmigrantes Latinoamericanos en España. Alicante: ECU, pp.15-26.

GARRIDO, L. (2008). "La inmigración en España". En: M. Requena y J. J. González (coord.). Tres décadas de cambio social en España. Madrid: Alianza Editorial, pp.127-164.

GONZÁLEZ RÍO, M. J. (1997). Métodos y técnicas de investigación social. Técnicas de recolección de datos. Alicante: Aguaclara.

GONZÁLEZ SANZ, G. (2011). "La primacía de lo local y lo privado en los británicos de la Comunidad Valenciana”. En: Más Poder Local, 7: 14-18.

HUETE, R. (2005). "Factores de atracción de Alicante como destino residencial: el punto de vista de los residentes europeos". En: Mazón, T. y Aledo, A. (coords.). Turismo residencial y cambio social. Nuevas perspectivas teóricas empíricas. Alicante: CAM, Fundación Frax y Universidad de Alicante, pp. 375-390.

NÚÑEZ, E. (2013). "Comunitat Valenciana: Una integración de vanguardia. La escuela de acogida". En: Barataria. Revista Castellano Manchega de Ciencias Sociales, 13: 75-86.

UÑA, O. y MARTÍN, A. (2009). Introducción a la Sociología. Madrid: Editorial Universitas.

Otras fuentes documentales consultadas:

INSTITUTO NACIONAL DE ESTADÍSTICA. http://www.ine.es/

MINISTERIO DE HACIENDA Y ADMINISTRACIONES PÚBLICAS. Secretaria de Estado de Presupuestos y Gastos. http://www.sepg.pap.minhap.gob.es/sitios/sepg/es-ES/Presupuestos/Presupuesto sEntidadesLocales/Paginas/PresupuestosEntidadesLocales.aspx OBSERVATORIO VALENCIANO DE LA INMIGRACIÓN.

Suma Gestión Tributaria de la Diputación Provincial de Alicante. https://www.suma.es/ 


\section{Breve currículo:}

\section{Irene Belmonte Martín}

Profesora del Área de Ciencia Política y de la Administración de la Universidad Miguel Hernández. Doctora por la Universidad de Alicante con el trabajo titulado: "Una estrategia de modernización administrativa en el ámbito local: El estudio de Suma Gestión Tributaria de la Diputación Provincial de Alicante (1990-2013)". Así, la línea de investigación principal es la Modernización de la Administración Pública, especialmente en lo que se refiere a la adaptación de la gestión de los ingresos públicos locales a la Gobernanza y al Nuevo Servicio Público. Otras líneas de trabajo, se encuadran en el análisis de las posibilidades de las NTICS aplicadas al gobierno, en la evaluación de las políticas públicas de igualdad, y el estudio de los Estados del Bienestar locales comparados. 